\title{
基于序列图像的深空小天体探测自主相对导航 误差补偿方法研究
}

\author{
侯博文, 王烔琦 ${ }^{*}$, 周海银, 周荁影, 何章鸣 \\ 国防科技大学文理学院, 长沙 410073 \\ *联系人, E-mail: wjq_gfkd@163.com \\ 收稿日期: 2020-12-10; 接受日期: 2021-02-04; 网络出版日期: 2021-07-08 \\ 国家自然科学基金(编号: U20B2055, 61525301, 61773021)和湖南省研究生创新项目(编号: CX20200037)资助
}

\begin{abstract}
摘要深空小天体是典型的空间非合作目标. 基于序列图像的空间非合作目标自主相对导航是实现小天体探测 的重要技术手段. 由于在轨环境复杂, 相机不可避免地会出现测量偏差, 进而影响相对导航状态估计精度. 本文在 充分利用相机视场内恒星序列图像的基础上, 通过最小二乘法计算相机在轨标定初值, 结合测量偏差状态转移模 型和“恒星对”之间的星光角距测量方程, 将测量偏差扩维成状态估计参数, 构建了基于相机在轨误差补偿的相对 导航状态估计模型. 结合无迹卡尔曼滤波算法, 通过序列图像的帧间信息对比进行迭代估计, 获得相机测量偏差 和相对导航系统状态，提高状态估计精度. 仿真分析中采用2016HO3小天体作为相对导航目标，仿真结果说明了 该方法的有效性, 能够有效地补偿相机测量偏差, 实现相对导航系统状态高精度估计, 为后续开展相关任务提供 了一定的技术支撑.
\end{abstract}

关键词小天体探测, 序列图像, 误差补偿, 恒星角距, 相对导航

PACS: 42.30.-d, 06.20.Dk, 95.10.Eg, 95.40.+s

\section{1 引言}

“天问一号”火星探测器的成功发射拉开了我国深 空探测的序幕，下一步将论证并逐步开展小天体探测 计划. 小天体是典型的空间非合作目标，对距离远、 非合作的小天体进行附着探测，依靠地面测控网进行 导航会极大降低导航的精度、可靠性及实时性 ${ }^{[1]}$.日 本“隼鸟”号探测器因地面通信延迟，未能准确掌握巡 视器释放窗口, 造成任务部分失败 ${ }^{[2]}$. 为了提高探测器
在各种未知环境下的适应能力, 必须发展小天体探测 全程自主相对导航技术. 在无法大幅度提高探测器星 上处理能力的约束下, 精简导航敏感器配置是实现小 天体探测全程自主相对导航的有效途径. 光学敏感器 质量小、功耗低, 作用距离远, 信息获取丰富, 是实现 小天体探测全程自主相对导航的理想手段 ${ }^{[3,4]}$.

小天体探测一般可分为巡航段、接近段和附着段 等几个典型的工作阶段. 在接近段, 探测器上的导航系 统就需要自主识别目标并确定相对位置和速度, 这是

引用格式: 侯博文, 王畑琦, 周海银, 等. 基于序列图像的深空小天体探测自主相对导航误差补偿方法研究. 中国科学: 物理学力学天文学, 2022, 52: 214507 Hou B W, Wang J Q, Zhou H Y, et al. Autonomous relative navigation error compensation method for small body exploration based on sequenceimages (in Chinese). Sci Sin-Phys Mech Astron, 2022, 52: 214507, doi: 10.1360/SSPMA-2020-0485 
实现全程自主相对导航的关键，也是实现小天体探测 的核心前提. 小天体是典型的空间非合作目标, 目前, 仅用光学序列图像的空间非合作目标自主相对导航技 术已经逐渐发展起来并应用于空间目标在轨服务中. 德国宇航中心在服务航天器轨道机动的基础上, 仅利 用光学图像信息实现了空间目标相对导航技术, 分析 了系统的可观测性 ${ }^{[5]}$, 并分别于 2012年和2016年将其 应用于PRISMA和AVANTI非合作目标交会对接试验 任务中 ${ }^{[6]}$; 斯图加特大学的Grzymisch和Fichter ${ }^{[7]}$ 给出 了使系统满足可观测性的轨道机动条件, 并设计了使 系统可观测性最优的轨道机动制导与控制方案 ${ }^{[8,9]}$. 上 述方法虽然都有效地提高了仅用光学序列图像的空间 非合作目标自主相对导航系统的可观测性, 为空间目 标在轨服务提供了有力的技术支撑, 但是并没有考虑 到光学敏感器的测量偏差对相对导航精度的影响. 通 常来讲, 敏感器会在地面进行精确标定, 但是航天器 发射过程中的抖动、在轨环境的热变化以及仪器老化 等影响会使敏感器测量基准出现偏差 ${ }^{[10]}$. 因此, 对光 学敏感器进行在轨误差校正对于提高相对导航精度具 有重要作用.

目前, 国内外研究学者大多采用多敏感器互补、 误差影响函数等方法实现误差补偿. Armellin等人 ${ }^{[1]}$ 在仅获得测角信息的情况下, 在初始轨道确定中利用 泰勒微分代数将误差从观测空间映射到状态空间, 通 过计算误差数据的均值和协方差实现误差补偿; 张春 青等人 ${ }^{[12]}$ 基于线性时变系统的可观测性理论将未知 系统偏差作为待估参数, 通过自校准滤波实现误差补 偿; Abdelrahman和Park ${ }^{[13]}$ 在航天器姿态动力学的基础 上, 利用磁强计和光纤陀螺仪测量数据实现了误差补 偿; Chang等人 ${ }^{[14]}$ 采用姿态数据库校准敏感器测量误 差; Wang等人 ${ }^{[15]}$ 提出了一种光学敏感器的在轨分布标 定方法, 该方法可在外部参数标定的广义相机框架下, 实现内部参数标定; 熊凯等人 ${ }^{[16-18]}$ 通过参数变换有效 地减少了误差待估参数数量, 实现了误差的估计和补 偿; Zhang等人 ${ }^{[19]}$ 在没有先验信息的情况下, 采用反向 神经网络和无迹卡尔曼滤波(Unscented Kalman Filter, UKF)算法分别对敏感器的多源系统误差进行粗校准 和精确校准, 具有很高的精度和较强的鲁棒性; Wang 等人 ${ }^{[20]}$ 采用傅里叶变换对敏感器的周期性误差进行 了补偿. 这些方法虽然都较好地实现了敏感器系统误 差校正, 但是由于系统待估参数增加, 在现有测量信
息的基础上，难以满足系统的可观测性，需要多敏感 器互校正或者采用智能算法实现误差参数估计, 增加 了导航系统的设备咒余度和计算复杂度, 难以星上 实现.

针对上述问题，本文在仅用单目相机进行小天体 探测自主相对导航过程中, 充分利用相机视场内的恒 星序列图像信息，基于恒星对角距正交变换不变的特 性构建了相机测量偏差测量方程, 并将测量偏差作为 相对导航系统待估参数, 通过构建新的相对导航系统, 估计出相机成像平面测量偏差和焦距误差参数, 进而 提高小天体相对位置状态估计精度.

\section{2 基于序列图像的小天体探测自主相对导 航模型}

\section{1 相对轨道动力学模型}

深空探测器在接近小天体时, 假设二者的轨道为 近圆轨道并且距离较近(远小于轨道半径), 小天体轨 道坐标系 $z$ 轴指向中心天体, $y$ 轴为轨道角速度方向的 反方向, $x$ 轴与 $y$ 轴、 $z$ 轴呈右手坐标系, 探测器轨道坐 标系与小天体轨道坐标系定义类似, 则以探测器轨道 坐标系为基准构建二者的相对运动模型, 可采用Clohessy-Wiltshire $(\mathrm{CW})$ 方程近似表示为

$\left\{\begin{array}{l}\ddot{x}(t)=2 \omega(t) \dot{z}(t)+a_{x}(t), \\ \ddot{y}(t)=-\omega^{2}(t) y(t)+a_{y}(t), \\ \ddot{z}(t)=-2 \omega(t) \dot{x}(t)+3 \omega^{2}(t) z(t)+a_{z}(t),\end{array}\right.$

其中 $(x(t), y(t), z(t))^{\mathrm{T}}$ 为小天体在探测器的轨道坐标系 中的位置向量, $t$ 为时间, 坐标系的中心为探测器的质 心, 探测器的轨道角速度为 $\omega(t)^{[21]} \cdot(\dot{x}(t), \dot{y}(t), \dot{z}(t))^{\mathrm{T}}$ 和 $(\ddot{x}(t), \ddot{y}(t), \ddot{z}(t))^{\mathrm{T}}$ 分别代表相对速度矢量和相对加速度 矢量, $\left(a_{x}(t), a_{y}(t), a_{z}(t)\right)^{\mathrm{T}}$ 代表轨道机动产生的瞬时加 速度. 为了简便, 将式(1)表示为

$\dot{\mathbf{x}}(t)=\mathbf{F}(t) \mathbf{x}(t)+\mathbf{B}(t) u(t)$,

其中 $\mathbf{x}(t)=(x(t), y(t), z(t), \dot{x}(t), \dot{y}(t), \dot{z}(t))^{\mathrm{T}}$ 代表小天体的 相对状态变量, $\mathbf{F}(t)$ 代表状态转移矩阵, $\mathbf{B}(t)$ 代表控制 量的输入矩阵, $u(t)$ 代表由机动使探测器状态改变的控 制加速度, 当机动形式为脉冲机动时, 在式(1)的前提 条件下, $\omega(t), \mathbf{F}(t)$ 以及 $\mathbf{B}(t)$ 为常值, 可以表示为 


$$
\begin{aligned}
\mathbf{F}(t) & =\left(\begin{array}{cccccc}
0 & 0 & 0 & 1 & 0 & 0 \\
0 & 0 & 0 & 0 & 1 & 0 \\
0 & 0 & 0 & 0 & 0 & 1 \\
0 & 0 & 0 & 0 & 0 & 2 \omega \\
0 & -\omega^{2} & 0 & 0 & 0 & 0 \\
0 & 0 & 3 \omega^{2} & -2 \omega & 0 & 0
\end{array}\right), \\
\mathbf{B}(t) & =\left(\begin{array}{ll}
\mathbf{O}_{3 \times 3} & \mathbf{I}_{3 \times 3}
\end{array}\right)^{\mathrm{T}},
\end{aligned}
$$

$$
\mathbf{u}(t)= \begin{cases}\left(a_{x}(t) a_{y}(t) a_{z}(t)\right)^{\mathrm{T}}, & t=t_{k}, \\ 0, & t \neq t_{k} .\end{cases}
$$

这里 $t_{k}$ 为脉冲机动时刻，则 $\mathrm{CW}$ 方程的离散形式可以表 示为 $^{[22]}$

$$
\mathbf{x}_{k}=\mathbf{\Phi} \mathbf{x}_{k-1}+\mathbf{G u} \text {, }
$$

其中,

$$
\boldsymbol{\Phi}=\left(\begin{array}{cccccc}
1 & 0 & 6 \omega T-6 \sin (\omega T) & \frac{4 \sin (\omega T)}{\omega}-3 T & 0 & \frac{4 \sin ^{2}(\omega T / 2)}{\omega} \\
0 & \cos (\omega T) & 0 & 0 & \frac{\sin (\omega T)}{\omega} & 0 \\
0 & 0 & 4-3 \cos (\omega T) & -\frac{4 \sin ^{2}(\omega T / 2)}{\omega} & 0 & \frac{\sin (\omega T)}{\omega} \\
0 & 0 & -6 \omega(\cos (\omega T)-1) & 4 \cos (\omega T)-3 & 0 & 2 \sin (\omega T) \\
0 & -n \sin (\omega T) & 0 & 0 & \cos (\omega T) & 0 \\
0 & 0 & 3 \omega \sin (\omega T) & -2 \sin (\omega T) & 0 & \cos (\omega T)
\end{array}\right),
$$

$$
\mathbf{G}=\left(\begin{array}{ccc}
\frac{4 \sin (\omega T)}{\omega}-3 T & 0 & \frac{4 \sin ^{2}(\omega T / 2)}{\omega} \\
0 & \frac{\sin (\omega T)}{\omega} & 0 \\
-\frac{4 \sin ^{2}(\omega T / 2)}{\omega} & 0 & \frac{\sin (\omega T)}{\omega} \\
4 \cos (\omega T)-3 & 0 & 2 \sin (\omega T) \\
0 & \cos (\omega T) & 0 \\
-2 \sin (\omega T) & 0 & \cos (\omega T)
\end{array}\right),
$$

$T$ 代表采样时间间隔，则 $\boldsymbol{\Phi}$ 和 $\mathbf{G}$ 分别为 $\mathbf{F}$ 和 $\mathbf{B}$ 的离散形 式, 且为常数矩阵.

\section{2 测量模型}

探测器采用单目相机作为测量设备，为了简化问 题研究, 本文假设相机坐标系与探测器轨道坐标系重 合, 相机测量原理如图1所示.

图1中 $\left(x_{0}, y_{0}\right)$ 为相机主点坐标, $f$ 为相机焦距, $O-x^{c} y^{c} z^{c}$ 为相机测量坐标系, $\left(z_{x}, z_{y}\right)$ 为小天体在探测 器相机像平面的坐标, $(x, y, z)$ 代表小天体在相机测量 坐标系中的坐标, 则在 $k$ 时刻, 相机测量模型为

$\widetilde{z_{k}}=\left(\begin{array}{c}z_{x_{k}}-x_{0} \\ z_{y_{k}}-y_{0}\end{array}\right)=\frac{f}{z_{k}}\left(\begin{array}{l}x_{k} \\ y_{k}\end{array}\right)+v_{\mathrm{c}}$,

其中 $v_{\mathrm{c}}$ 为相机测量噪声, 且满足 $\mathrm{E}\left(v_{\mathrm{c}}\right)=0, \mathrm{E}\left(\mathbf{v}_{\mathrm{c}}^{\mathrm{T}} \mathbf{v}_{\mathrm{c}}\right)=\mathbf{R}_{\mathrm{c}}, \mathbf{R}_{\mathrm{c}}$ 为测量噪声方差矩阵. 然而, 测量模型(9)无法提供距
离信息, 难以实现相对导航系统状态完备估计. 而轨 道机动是一种提高系统可观测度的有效方法，根据探 测器的轨道机动控制量可以得到机动距离, 进而提高 系统可观测度. 针对轨道机动量对系统可观测度影响 的分析, 可以参考文献[8], 这里不做赘述.

如图2所示, $\mathbf{r}_{0, k}$ 和 $\mathbf{r}_{0, k}$ 分别代表探测器机动前和机 动后的小天体相对位置向量, 由于相机成像平面相对 于小天体位置是个很小的量，可以忽略不计，因此可 以用 $\mathbf{r}_{k}$ 和 $\mathbf{r}_{k}$ 来代替 $\mathbf{r}_{0, k}$ 和 $\mathbf{r}_{0, k} . \delta \mathbf{r}_{k}$ 代表轨道机动向量. 根 据正弦定理, 则在 $k$ 时刻有如下几何关系:

$\mathbf{r}_{k}=\frac{\sin \beta_{k}}{\sin \alpha_{k}} \delta \mathbf{r}_{k}$,

其中 $\alpha_{k}$ 代表矢量 $\mathbf{r}_{k}$ 和 $\mathbf{r}_{k}$ 的夹角, $\beta_{k}$ 代表矢量 $\delta \mathbf{r}_{k}$ 和 $\mathbf{r}_{k}$ 的夹 角. 则两个夹角可以计算如下:

$\alpha_{k}=\arccos \left(\mathbf{r}_{k} \cdot \mathbf{r}_{k} /\left|\mathbf{r}_{k}\right|\left|\mathbf{r}_{k}\right|\right)$,

$\beta_{k}=\arccos \left(\delta \mathbf{r}_{k} \cdot \widetilde{\mathbf{r}}_{k} /\left|\delta \mathbf{r}_{k}\right|\left|\widetilde{\mathbf{r}}_{k}\right|\right)$.

根据式(6)，假设在 $k-1$ 时刻执行轨道机动，则 $\mathbf{r}_{k}$ 和 $\mathbf{r}_{k}$ 计算如下:

$$
\begin{aligned}
\widetilde{\mathbf{r}}_{k} & =\boldsymbol{\Phi}_{1} \mathbf{x}_{k-1}, \\
\mathbf{r}_{k} & =\boldsymbol{\Phi}_{1} \mathbf{x}_{k-1}+\mathbf{G}_{1} \mathbf{u} .
\end{aligned}
$$

进而结合式(10), 可以得到在 $k$ 时刻机动后位置与 机动前位置的距离: 


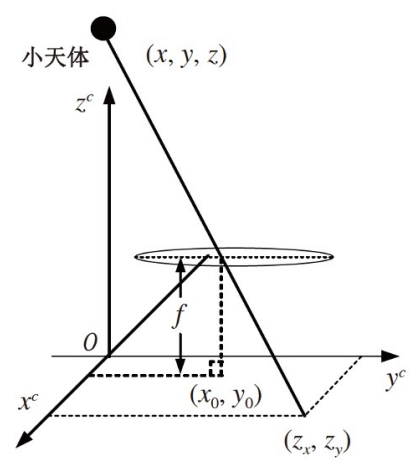

图 1 相机测量原理

Figure 1 Camera measurement principle.

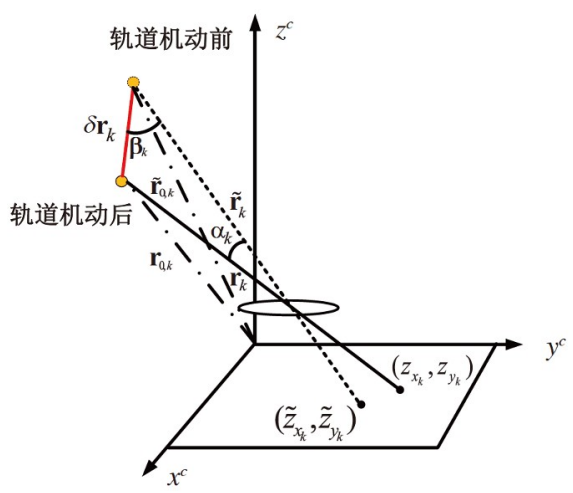

图 2 (网络版彩图)轨道机动示意图

Figure 2 (Color online) Orbit maneuvering diagram.

$d_{k}=\left|\mathbf{r}_{k}\right|=\left|\mathbf{G}_{1} \mathbf{u}\right|=\left|\delta \mathbf{r}_{k} \sin \beta_{k} / \sin \alpha_{k}\right|$,

其中 $\boldsymbol{\Phi}_{1}$ 分别代表矩阵 $\boldsymbol{\Phi}$ 的前三行元素构成的矩阵, $\mathbf{G}_{1}$ 代表矩阵 $\mathbf{G}$ 的前三行元素构成的矩阵. 进一步, 在进 行脉冲机动后第 $n$ 个时刻, 有机动和无机动情况下, 目 标外推位置的距离表示如下:

$d_{k+n}=\left|\boldsymbol{\Phi}_{1}^{n} \mathbf{G}_{1} \mathbf{u}\right|=\left|\delta \mathbf{r}_{k+n} \sin \beta_{k+n} / \sin \alpha_{k+n}\right|$.

因此，式(16)可作为距离测量信息，但由于相机本 身存在随机测量误差，因此该距离测量信息也存在随 机误差，文献[23]对此作了详细分析，这里不做赘述， 其标准差为

$\sigma_{d_{k}}=\sigma_{\mathrm{c}}\left|\mathbf{r}_{k}\right| \cot \alpha_{k}$,

其中 $\sigma_{\mathrm{c}}$ 为相机随机测量误差的标准差.

根据式(9)，(10)和(15) 可以得到在 $k$ 时刻的测量 方程: $\mathbf{z}_{k}=\left(\begin{array}{c}z_{x, k}-x_{0} \\ z_{y, k}-y_{0} \\ d_{k}\end{array}\right)=\left(\begin{array}{c}\frac{f x_{k}}{z_{k}} \\ \frac{f y_{k}}{z_{k}} \\ \sqrt{x_{k}^{2}+y_{k}^{2}+z_{k}^{2}}\end{array}\right)+\left(\begin{array}{c}v_{c, k} \\ v_{c, k} \\ v_{R, k}\end{array}\right)$,

其中 $v_{R, k}$ 代表距离测量值的随机误差, $\mathrm{E}\left(\mathbf{v}_{R, k}^{\mathrm{T}} v_{R, k}\right)=\sigma_{d_{k}}^{2}$. 该方程被称为伪测量方程，因此距离信息并非真实测 量得到, 而是通过轨道机动和相对轨道外推获得的.

\section{3 基于误差在轨校正的自主相对导航方法}

当相机受到振动、热效应等影响时，相机的焦距 和主点位置会存在常值误差 $\Delta \mathbf{x}=\left(\Delta x_{0}, \Delta y_{0}, \Delta f\right)^{\mathrm{T}}$, 此时 会对相对导航状态估计结果造成较大影响. 为了克服 相机测量常值偏差造成的影响, 有必要对相机测量偏 差进行在轨补偿并实现高精度状态估计.

\section{1 基于状态扩维的误差校正状态模型}

为了能实时地估计常值偏差并且实现误差校正, 需要将误差变量作为待估参数, 并将其与原相对导航 状态方程(6)组合成一个新的状态模型如下:

$\widehat{\mathbf{x}}_{k}=\left(\begin{array}{c}\mathbf{x}_{k} \\ \Delta \mathbf{x}_{k}\end{array}\right)=\left(\begin{array}{cc}\mathbf{\Phi} & \mathbf{O}_{3 \times 3} \\ \mathbf{O}_{3 \times 3} & \mathbf{I}_{3 \times 3}\end{array}\right)\left(\begin{array}{c}\mathbf{x}_{k-1} \\ \Delta \mathbf{x}_{k-1}\end{array}\right)+\left(\begin{array}{c}\mathbf{G u} \\ \mathbf{O}_{3 \times 1}\end{array}\right)+\boldsymbol{\omega}_{k}$,

其中 $\widetilde{\mathbf{x}}_{k}=\left(\begin{array}{ll}\mathbf{x}_{k} & \Delta \mathbf{x}_{k}\end{array}\right)^{\mathrm{T}}$ 为新的状态变量, $\boldsymbol{\omega}_{k}$ 代表高斯白 噪声, 且有 $\mathrm{E}\left(\boldsymbol{\omega}_{k}^{\mathrm{T}} \boldsymbol{\omega}_{k}\right)=\mathbf{Q}_{k}, \mathbf{Q}_{k}$ 为噪声方差. $\mathbf{I}_{3 \times 3}$ 为 $3 \times 3$ 的 单位矩阵.

\section{2 误差校正方法}

探测器相机成像获得的不只有小天体, 还有视场 内的其他恒星, 如图3所示.

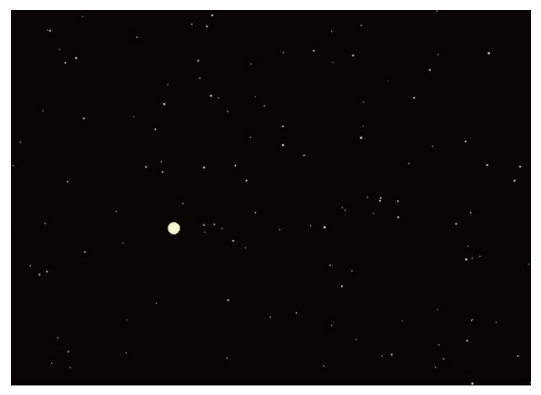

图 3 (网络版彩图)相机视场内的目标图像

Figure 3 (Color online) Target images in the camera field of view. 
图中白色圆点代表目标小天体, 其余星点为被观 测恒星. 恒星在相机平面内测量原理与图1类似, 则恒 星在惯性系下星光矢量 $\mathbf{s}_{\mathrm{i}}$ 与测量系下星光矢量 $\mathbf{s}_{\mathrm{c}}$ 存在 如下关系:

$\mathbf{s}_{\mathrm{c}}=\mathbf{M} \mathbf{s}_{\mathrm{i}}$,

其中 $\mathbf{M}$ 为相机的姿态矩阵, $\mathbf{s}_{\mathrm{i}}$ 和 $\mathbf{s}_{\mathrm{c}}$ 分别表示如下:

$\mathbf{s}_{\mathrm{i}}=\left(\begin{array}{c}\cos \alpha \cos \delta \\ \sin \alpha \cos \delta \\ \sin \delta\end{array}\right)$,

$\mathbf{s}_{\mathrm{c}}=\frac{1}{\sqrt{\left(s_{x}-x_{0}\right)^{2}+\left(s_{y}-y_{0}\right)^{2}+f^{2}}}\left(\begin{array}{c}-\left(s_{x}-x_{0}\right) \\ -\left(s_{y}-y_{0}\right) \\ f\end{array}\right)$.

$(\alpha, \delta)$ 为恒星在天球坐标系下的赤经赤纬, $\left(s_{x}, x_{y}\right)$ 为 恒星在相机像平面的坐标, 与 $\left(z_{x}, z_{j y}\right)$ 含义类似. 在相 机视场内有多个恒星, 假设恒星 $s_{p}$ 和 $s_{q}$ 的夹角为 $\theta_{p q}$, 则有

$\cos \theta_{p q}=\mathbf{s}_{\mathrm{c}}^{\mathrm{T}} \mathbf{s}_{\mathrm{c}}=\mathbf{s}_{\mathrm{i}}^{\mathrm{T}} \mathbf{M}^{\mathrm{T}} \mathbf{M} \mathbf{s}_{\mathrm{i}}=\mathbf{s}_{\mathrm{i}}^{\mathrm{T}} \mathbf{s}_{\mathrm{i}}$.

在考虑相机噪声的情况下, 式(23)可以表示成

$\mathbf{s}_{\mathrm{i}}^{\mathrm{T}} \mathbf{s}_{\mathrm{i}}=\mathbf{s}_{\mathrm{c}}^{\mathrm{T}} \mathbf{s}_{\mathrm{c}}+\mathbf{v}_{\mathrm{c}}$.

将式(21)和(22)带入到式(24)中, 则有

$\mathbf{s}_{\mathrm{i}}^{\mathrm{T}} \mathbf{s}_{\mathrm{i}}=\frac{D}{S_{P} S_{2}}+v_{\mathrm{c}}=l_{p q}\left(x_{0}, y_{0}, f\right)+\mathbf{v}_{\mathrm{c}}$

其中

$\left\{\begin{array}{l}D=\left(s_{p x}-x_{0}\right)\left(s_{q x}-x_{0}\right)+\left(s_{p y}-y_{0}\right)\left(s_{q y}-y_{0}\right)+f^{2}, \\ S_{1}=\sqrt{\left(s_{p x}-x_{0}\right)^{2}+\left(s_{p y}-y_{0}\right)+f^{2}}, \\ S_{2}=\sqrt{\left(s_{q x}-x_{0}\right)^{2}+\left(s_{q y}-y_{0}\right)+f^{2}},\end{array}\right.$

$\mathbf{v}_{\mathrm{c}}=\left(\begin{array}{llll}v_{c, k} & v_{c, k} & v_{c, k}\end{array}\right)^{\mathrm{T}}$,

并且有

$l_{p q}\left(x_{0}, y_{0}, f\right)=\frac{D}{S_{1} S_{2}}$.

当相机存在常值偏差时, 则有

$\left\{\begin{array}{l}x_{0}=\widehat{x}_{0}+\Delta x \\ y_{0}=\widehat{y}_{0}+\Delta y \\ f=\widehat{f}+\Delta f\end{array}\right.$
其中 $\left(\widehat{x}_{0}, \widehat{y}_{0}, \widehat{f}\right)^{\mathrm{T}}$ 代表偏差估计值, 将式(29)代入式(25), 则有

$\mathbf{s}_{\mathrm{i}}^{\mathrm{T}} \mathbf{s}_{\mathrm{i}}=l_{p q}\left(\widehat{x}_{0}, \widehat{y}_{0}, \widehat{f}\right)+\left(\begin{array}{lll}\frac{\partial l_{p q}}{\partial x_{0}} & \frac{\partial l_{p q}}{\partial y_{0}} & \frac{\partial l_{p q}}{\partial f}\end{array}\right)\left(\begin{array}{c}\Delta x_{0} \\ \Delta y_{0} \\ \Delta f\end{array}\right)+\mathbf{v}_{\mathrm{c}}$,

令

$\mathbf{E}_{p q}=\mathbf{s}_{\mathrm{i}}^{\mathrm{T}} \mathbf{s}_{\mathrm{i}}-l_{p q}\left(\widehat{x}_{0}, \widehat{y}_{0}, \widehat{f}\right)=\mathbf{L}_{p q}\left(\begin{array}{c}\Delta x_{0} \\ \Delta y_{0} \\ \Delta f\end{array}\right)+\mathbf{v}_{\mathrm{c}}$,

其中

$\mathbf{L}_{p q}=\left(\begin{array}{lll}\frac{\partial l_{p q}}{\partial x_{0}} & \frac{\partial l_{p q}}{\partial y_{0}} & \frac{\partial l_{p q}}{\partial f}\end{array}\right)$.

假设视场内的恒星数量为 $n$, 则式(31)可以写作:

$\mathbf{E}=\mathbf{L}\left(\Delta x_{0} \Delta y_{0} \Delta z_{0}\right)^{\mathrm{T}}+\mathbf{V}_{\mathrm{c}}$,

其中

$\mathbf{L}=\left(\begin{array}{ccc}\frac{\partial l_{12}}{\partial x_{0}} & \frac{\partial l_{12}}{\partial y_{0}} & \frac{\partial l_{12}}{\partial f} \\ \frac{\partial l_{13}}{\partial x_{0}} & \frac{\partial l_{13}}{\partial y_{0}} & \frac{\partial l_{13}}{\partial f} \\ \vdots & \vdots & \vdots \\ \frac{\partial l_{p q}}{\partial x_{0}} & \frac{\partial l_{p q}}{\partial y_{0}} & \frac{\partial l_{p q}}{\partial f} \\ \vdots & \vdots & \vdots \\ \frac{\partial l_{(n-1) n}}{\partial x_{0}} & \frac{\partial l_{(n-1) n}}{\partial y_{0}} & \frac{\partial l_{(n-1) n}}{\partial f}\end{array}\right), \mathbf{E}=\left(\begin{array}{c}\mathbf{E}_{12} \\ \mathbf{E}_{13} \\ \vdots \\ \mathbf{E}_{p q} \\ \vdots \\ \mathbf{E}_{(n-1) n}\end{array}\right)$,

这里 $p=1,2, \ldots,(n-1), q=p+1, \ldots, n ; \mathbf{E}$ 代表测量噪声 矩阵.

最小二乘法是实现相机测量偏差向量 $\left(\Delta x_{0}, \Delta y_{0}, \Delta f\right)^{\mathrm{T}}$ 估计的最佳途径之一 ${ }^{[23]}$, 但是在测量噪声影响下, 视 场内星光角距测量值会被淹没, 状态估计结果不稳定; 为了提高抗干扰性, 有必要引入常值偏差的状态转移 方程进行滤波估计, 进一步提高常值偏差状态量的估 计精度, 从而降低其对自主相对导航状态估计精度的 影响.

\section{3 结合误差在轨校正的自主相对导航方法}

结合相对导航状态和测量模型, 可以得到基于误 
差在轨自主校正的自主相对导航模型如下:

$$
\left\{\begin{array}{l}
\tilde{\mathbf{x}}_{k}=\left(\begin{array}{cc}
\boldsymbol{\Phi} & \mathbf{O}_{3 \times 3} \\
\mathbf{O}_{3 \times 3} & \mathbf{I}_{3 \times 3}
\end{array}\right)\left(\begin{array}{c}
\mathbf{x}_{k-1} \\
\Delta \mathbf{x}_{k-1}
\end{array}\right)+\left(\begin{array}{c}
\mathbf{G u} \\
\mathbf{O}_{3 \times 1}
\end{array}\right)+\boldsymbol{\omega}_{k}, \\
\widetilde{\mathbf{z}}_{k}=h\left(\widetilde{\mathbf{x}}_{k}\right)+\mathbf{e}_{k},
\end{array}\right.
$$

其中

$$
\widehat{\mathbf{z}}_{k}=\left(\begin{array}{ll}
\mathbf{z}_{k} & \mathbf{E}_{p q, k}
\end{array}\right)^{\mathrm{T}},
$$

$$
h\left(\hat{\mathbf{x}}_{k}\right)=\left(\begin{array}{c}
\frac{f x_{k}}{z_{k}} \\
\frac{f y_{k}}{z_{k}} \\
x_{k}^{2}+y_{k}^{2}+z_{k}^{2} \\
\mathbf{L}_{p q}\left(\Delta x_{0} \Delta y_{0} f\right)^{\mathrm{T}}
\end{array}\right), \mathbf{e}_{k}=\left(\begin{array}{c}
\mathbf{v}_{c, k} \\
\mathbf{v}_{c, k} \\
\mathbf{v}_{R, k} \\
\mathbf{v}_{c, k}
\end{array}\right) .
$$

在模型(35)的基础上利用UKF算法可以实现状态 估计 ${ }^{[24]}$ ，但是如果初始值误差较大会导致噪声滤波发 散，状态估计精度降低，因此有必要采用最小二乘法 先给出一个相机偏差的初始值作为UKF算法的输入. 具体流程如图4所示.

在图4中，Kalman滤波算法可以为测量偏差提供 状态转移信息，而且在滤波后增加连续两个时刻的序 列图像间偏差状态估计比较环节，可以有效提高参数 估计的稳定性. 另外，在利用最小二乘法估计偏差状 态时，一次估计不一定能够准确地计算出偏差状态估 计量，需要利用连续多个时刻的星图数据，即序列星 图数据, 才可获得稳定的偏差状态估计量. 而增加Kalman滤波算法估计状态偏差可以加快偏差状态估计效 率, 减少计算步骤.

\section{4 仿真验证}

本文选用太阳系小行星2016HO3为目标，小行星 探测阶段为接近段, 采用STK二体轨道模型仿真模型 进行仿真，星图数据来源于依巴谷星表仿真恒星，利 用不同时刻探测器获取的图像信息，选取视场内恒星

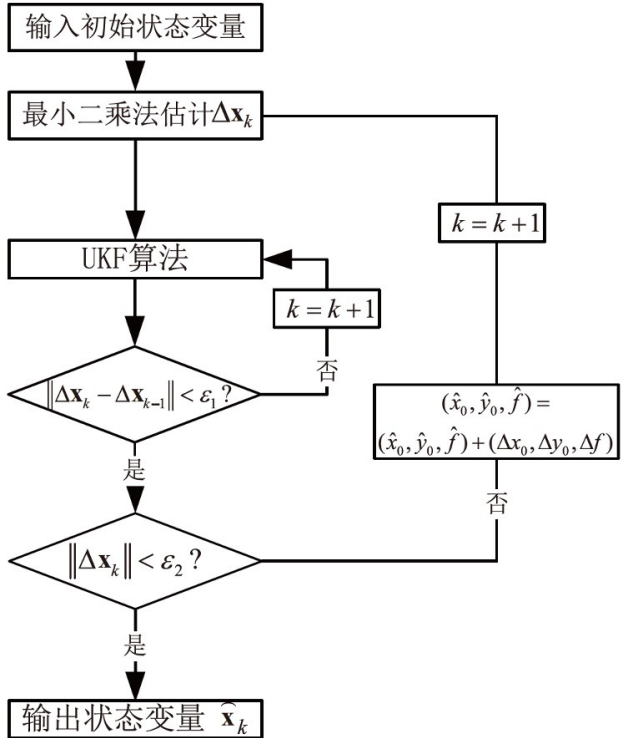

图 4 基于误差在轨校正的自主相对导航流程

Figure 4 Flowchart of the autonomous relative navigation based on the on-orbit calibration.

进行误差补偿. 探测器的轨道机动量为 $\left[\begin{array}{lll}19.94 & 2 & 3\end{array}\right]^{\mathrm{T}}$, 相对导航机动时刻为第 $10 \mathrm{~s}$, 仿真总时长为 $10^{6} \mathrm{~s}$, 误差 参数估计过程中采用的迭代阈值分别为 $\varepsilon_{1}=10^{-15}$, $\varepsilon_{2}=5 \times 10^{-6}$, 探测器和小行星在太阳系的轨道根数如表 1所示.

假设光学相机实际焦距为 $75 \mathrm{~mm}$ ，地面标定值为 $79 \mathrm{~mm}$ ，主点实际坐标为 $(50.00,50.00) \mathrm{mm}$ ，地面标定 值为 $(50.10,50.22) \mathrm{mm}$, 相机分辨率为 $1024 \times 1024$ 像素, 每个像素大小为 $10 \mu \mathrm{m} \times 10 \mu \mathrm{m}$ ，相机采样频率为 $1 \mathrm{~Hz}$, 测量标准差为 $1 \times 10^{-5} \mathrm{rad}$, 相对轨道如图 5 所示.

为了说明算法的稳定性，在没有Kalman滤波估计 的情况下, 测量偏差状态参数估计结果如图6所示.

在未进行在轨自主标定时，相对导航状态估计误 差如图7所示，相机测量偏差估计值如图8所示，在相 机自主标定后的相对导航状态估计误差如图9所示. 相机标定前后相对位置估计的均方误差(Root Mean Square Error, RMSE)如表2所示.

表 1 小行星和探测器轨道根数

Table 1 Orbit elements of the asteroid and the probe

\begin{tabular}{ccccccc}
\hline 项目 & 半长轴 $(\mathrm{km})$ & 偏心率 & 倾角 $\left(^{\circ}\right)$ & 近地点幅角 $\left(^{\circ}\right)$ & 升交点赤经 $\left(^{\circ}\right)$ & 平近点角 $\left(^{\circ}\right)$ \\
\hline 小行星 & $1.498 \times 10^{8}$ & 0.104 & 7.776 & 306.806 & 66.359 & 168.424 \\
探测器 & $1.498 \times 10^{8}$ & 0.050 & 7.000 & 300.000 & 66.000 & 170.000 \\
\hline
\end{tabular}




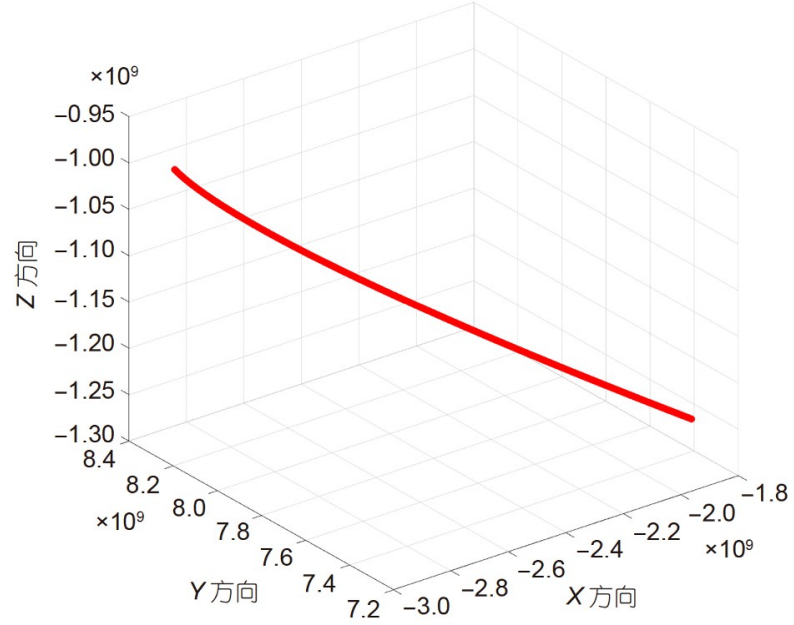

图 5 (网络版彩图)相对轨道示意图

Figure 5 (Color online) The diagram of the relative orbit.
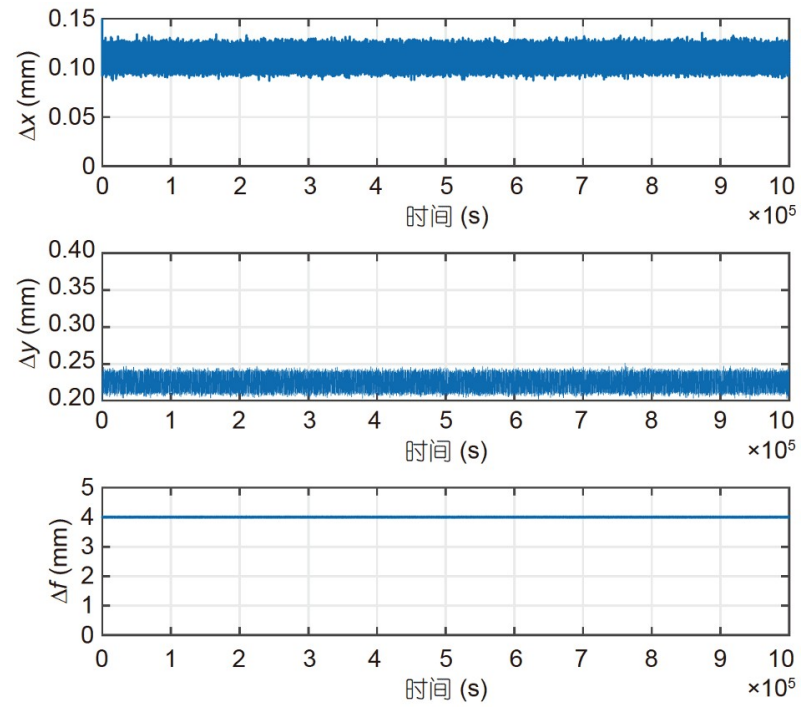

图 6 (网络版彩图)基于最小二乘法的测量偏差量状态估计值 Figure 6 (Color online) Measurement bias estimation results using least squares method.

对比图6和8可知，基于Kalman滤波的测量偏差量 状态估计结果更为稳定，主要是因为事先已知测量偏 差为常值偏差，可利用状态转移方程提高偏差量状态 估计的稳定性. 另外，根据图7-9，采用UKF算法将测 量偏差量作为系统待估参数可以直接将相对导航位置 状态和偏差量同时估计出来，避免了先估计偏差后估 计相对导航状态的繁琐流程.

由图7-9和表2可知，相机测量偏差对相对导航系 统状态估计的影响很大，这主要是因为在接近段探测
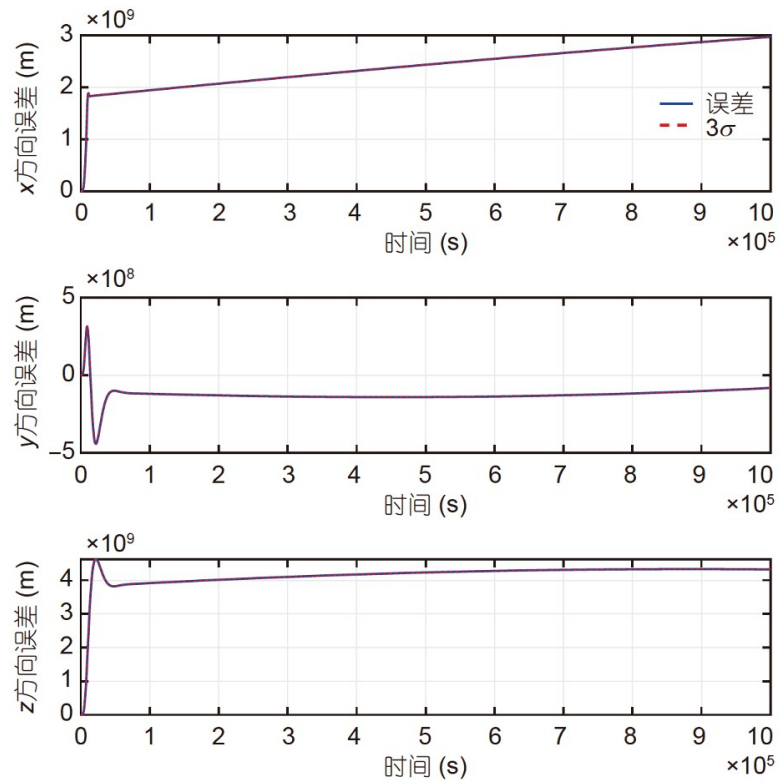

图 7 (网络版彩图)相机标定前相对导航状态估计误差示 意图

Figure 7 (Color online) Relative navigation state estimation error before camera calibration.
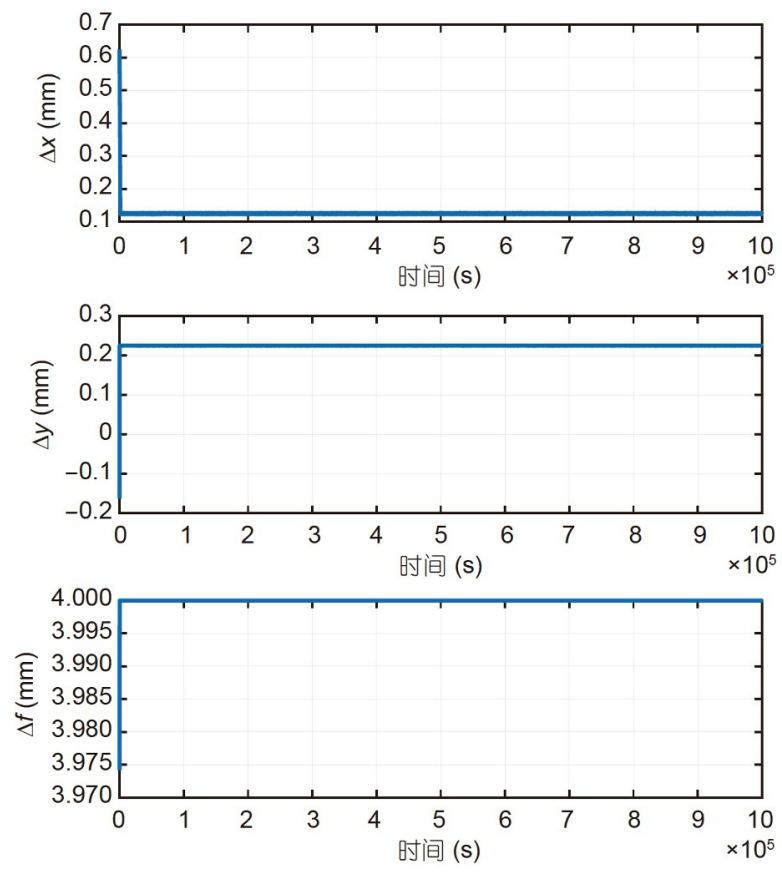

图 8 (网络版彩图)测量偏差量状态估计值

Figure 8 (Color online) Measurement bias state estimation.

器与小天体距离较远, 并且在仅用单目相机进行测量 的情况下，较小的测量偏差都会对状态估计产生非常 

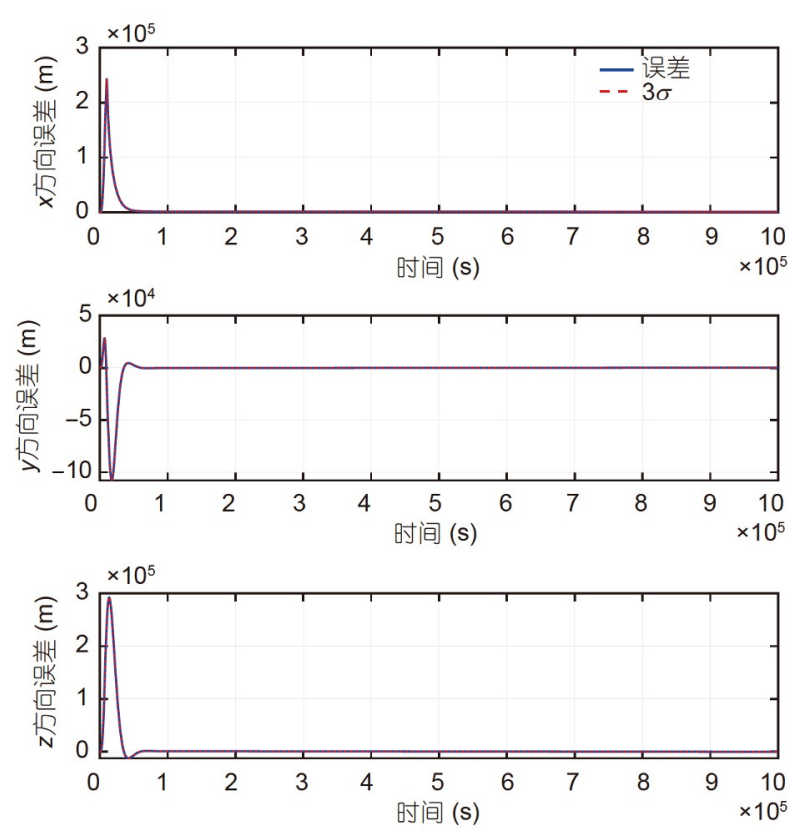

图 9 (网络版彩图)相机标定后相对导航状态估计误差示 意图

Figure 9 (Color online) Relative navigation state estimation error after camera calibration.

大的影响; 利用相机在轨误差补偿模型可以有效地估 计测量偏差, 并在此基础上实现相对导航状态估计,
表 2 相对导航状态估计均方误差比较

Table 2 Relative navigation state estimation RMSE comparison

\begin{tabular}{cccc}
\hline 状态 & $x$ 方向 $(\mathrm{m})$ & $y$ 方向 $(\mathrm{m})$ & $z$ 方向 $(\mathrm{m})$ \\
\hline 标定前 & $1.0859 \times 10^{9}$ & $7.4930 \times 10^{9}$ & $3.8663 \times 10^{9}$ \\
标定后 & $1.9549 \times 10^{4}$ & $2.8992 \times 10^{4}$ & $5.1018 \times 10^{4}$ \\
\hline
\end{tabular}

状态估计结果收玫性较好, 估计精度有很大的提高.

\section{5 结论}

本文以深空探测器小天体探测为背景, 针对单目 相机测量偏差带来的相对导航状态估计精度差的问 题, 开展了基于序列图像的相对导航误差补偿方法 研究. 充分利用相机视场内的恒星序列图像, 构建 结合相机在轨标定的小天体探测自主相对导航模型, 实现了相机测量偏差的在轨估计, 有效地提高了相对 导航状态估计精度. 该方法为单目相机相对导航状 态估计提供了一定的技术支撑，但是算法的迭代过程 会增加计算复杂度, 对于在轨资源严重受限的航天器 来说是一个函待解决的难题, 后续将围绕降低待估参 数维度实现相机标定和状态估计开展相应的研究 工作.

\section{参考文献}

1 Wang D Y, Huang X Y. Autonomous optical navigation for deep space transfer phase and its simulation verification (in Chinese). Contr Theory Appl, 2014, 31: 1714-1722 [王大轶, 黄翔宇. 深空探测转移段光学成像测量自主导航及仿真验证技术. 控制理论与应用, 2014, 31: 17141722]

2 Nakatani T Y T K I. Operation of MINERVA rover in Hayabusa asteroid mission. In: Proceedings of the 57th International Astronautical Congress. Valenica, 2006

3 Wang D Y, Zhang L, Zhu W H, et al. Autonomous relative navigation for noncooperative target using stereo vision measurements (in Chinese). Sci Sin-Phys Mech Astron, 2019, 49: 024509 [王大轶, 张否, 朱卫红, 等. 基于双目光学图像信息的空间非合作目标自主相对导航方法. 中国 科学: 物理学, 力学, 天文学, 2019, 49: 024509]

4 Kominato T, Matsuoka M, Uo M, et al. Optical hybrid navigation and station keeping around Itokawa. In: Proceedings of the AIAA/AAS Astrodynamics Specialist Conference and Exhibit. 2006. 6535

5 Gaias G, D’Amico S, Ardaens J S. Angles-only navigation to a noncooperative satellite using relative orbital elements. J Guid Control Dyn, 2014, 37: 439-451

6 Ardaens J S, Gaias G. Angles-only relative orbit determination in low earth orbit. Adv Space Res, 2018, 61: 2740-2760

7 Grzymisch J, Fichter W. Analytic optimal observability maneuvers for in-orbit bearings-only rendezvous. J Guid Control Dyn, 2014, 37: 1658- 
1664

8 Grzymisch J, Fichter W, Losa D, et al. Bearings-only Rendezvous with Enhanced Performance. In: Advances in Aerospace Guidance, Navigation and Control. Cham: Springer, 2015. 571-590

9 Grzymisch J, Fichter W. Optimal rendezvous guidance with enhanced bearings-only observability. J Guid Control Dyn, 2015, 38: 1131-1140

10 Medaglia E. Autonomous on-orbit calibration of a star tracker. In: Proceedings of the 2016 IEEE Metrology for Aerospace (MetroAeroSpace). New York: IEEE, 2016. 456-461

11 Armellin R, Di Lizia P, Zanetti R. Dealing with uncertainties in angles-only initial orbit determination. Celest Mech Dyn Astr, 2016, 125: 435450

12 Zhang C Q, Li Y, Liu L D, et al. A self-calibration filter for satellites autonomous orbit determination systems (in Chinese). J Aeronaut, 2006, 27: 301-305 [张春青, 李勇, 刘良栋, 等. 卫星自主轨道确定的自校准滤波. 宇航学报, 2006, 27: 301-305]

13 Abdelrahman M, Park S Y. Sigma-point Kalman filtering for spacecraft attitude and rate estimation using magnetometer measurements. IEEE Trans Aerosp Electron Syst, 2011, 47: 1401-1415

14 Chang J, Geng Y, Guo J, et al. Calibration of satellite autonomous navigation based on attitude sensor. J Guid Control Dyn, 2017, 40: 185-192

15 Wang M, Cheng Y, Yang B, et al. On-orbit calibration approach for optical navigation camera in deep space exploration. Opt Express, 2016, 24: $5536-5554$

16 Xiong K, Wei C L, Liu L D. Multiple-model adaptive estimator for attitude sensor calibration (in Chinese). J Syst Sci Math Sci, 2015, 35: 745756 [熊凯, 魏春岭, 刘良栋. 基于多模型自适应估计的姿态敏感器误差校准. 系统科学与数学, 2015, 35: 745-756]

17 Wei C L, Zhang B, Zhang C Q. An attitude maneuvering aided self-calibration algorithm for celestial autonomous navigation system (in Chinese). J Aeronaut, 2010, 31: 93-97 [魏春岭, 张斌, 张春青. 一种姿态机动辅助下的天文导航系统偏差自校准方法. 宇航学报, 2010, 31: 93-97]

18 Xiong K, Zong H. Performance evaluation of star sensor low frequency error calibration. Acta Astronaut, 2014, 98: 24-36

19 Zhang H, Niu Y, Lu J, et al. On-orbit calibration for star sensors without priori information. Opt Express, 2017, 25: 18393-18409

20 Wang J, Xiong K, Zhou H. Low-frequency periodic error identification and compensation for star tracker attitude measurement. Chin J Aeronaut, 2012, 25: 615-621

21 Li J R, Li H Y, Tang G J, et al. Research on the strategy of angles-only relative navigation for autonomous rendezvous. Sci China Tech Sci, 2011, 54: 1865-1872 [李九人, 李海阳, 唐国金, 等. 基于仅测角的自主交会相对导航策略研究. 中国科学: 技术科学, 2011, 41: 1197-1204]

22 Franquiz F J, Muñoz J D, Udrea B, et al. Optimal range observability maneuvers of a spacecraft formation using angles-only navigation. Acta Astronaut, 2018, 153: 337-348

23 Liu H B, Wang J Q, Tan J C, et al. Autonomous on-orbit calibration of a star tracker camera. Opt Eng, 2011, 50: 023604

24 Julier S J, Uhlmann J K. Unscented filtering and nonlinear estimation. Proc IEEE, 2004, 92: 401-422 


\title{
Autonomous relative navigation error compensation method for small body exploration based on sequence-images
}

\author{
HOU BoWen, WANG JiongQi ${ }^{*}$, ZHOU HaiYin, ZHOU XuanYing \& HE ZhangMing \\ College of Liberal Arts and Sciences, National University of Defense Technology, Changsha 410073, China
}

\begin{abstract}
Deep-space small bodies are typical space non-cooperative targets. The autonomous relative navigation of space noncooperative targets based on sequence-images is an important technical method to realize the small body exploration. Due to the complex on-orbit environment, it is inevitable that the camera will suffer the measurement deviations and the focal length deviation, which will severely affect the relative navigation state estimation accuracy. On the basis of making full use of the information of star sequence images in the field of view of the camera, the initial value of the camera onorbit calibration is calculated by the least square method. Combined with the measurement deviation state transition model and the starlight angular distance measurement equation between two stars, the measurement deviations are expanded into state estimation parameters and the relative navigation state estimation model based on camera on-orbit error compensation is constructed. Combined with the unscented Kalman filter algorithm, the iterative estimation is carried out through comparing the information between the frames of sequence images. Then the camera measurement deviation and the relative navigation system state are obtained. In this way, the state estimation accuracy is improved. In the simulation analysis, the small body $2016 \mathrm{HO} 3$ is considered as the relative navigation target. The simulation results show the effectiveness of the method, which can effectively compensate the camera measurement deviation and achieve the high-precision estimation of the relative navigation system state. The research results provide certain technical support for the subsequent related tasks.
\end{abstract}

small body exploration, sequence-images, error compensation, stellar angular distance, relative navigation

PACS: 42.30.-d, 06.20.Dk, 95.10.Eg, 95.40.+s

doi: 10.1360/SSPMA-2020-0485 